\title{
High-performance planar-type electron source based on a graphene-oxide- semiconductor structure
}

Cite as: Appl. Phys. Lett. 114, 213501 (2019); https://doi.org/10.1063/1.5091585

Submitted: 04 February 2019. Accepted: 09 May 2019. Published Online: 30 May 2019

Katsuhisa Murakami (D), Joji Miyaji, Ryo Furuya, Manabu Adachi, Masayoshi Nagao (D), Yoichiro Neo, Yoshinori Takao (D), Yoichi Yamada (D), Masahiro Sasaki, and Hidenori Mimura

ARTICLES YOU MAY BE INTERESTED IN

Near-infrared electroluminescence from metal-oxide-semiconductor devices with erbiumdoped gadolinium oxide on silicon

Applied Physics Letters 114, 211102 (2019); https://doi.org/10.1063/1.5084016

Sub-nanosecond switching in a cryogenic spin-torque spin-valve memory element with a dilute permalloy free layer

Applied Physics Letters 114, 212402 (2019); https://doi.org/10.1063/1.5094924

Atomic layer deposition of energy band tunable tin germanium oxide electron transport layer for the SnS-based solar cells with $400 \mathrm{mV}$ open-circuit voltage

Applied Physics Letters 114, 213901 (2019); https://doi.org/10.1063/1.5098766
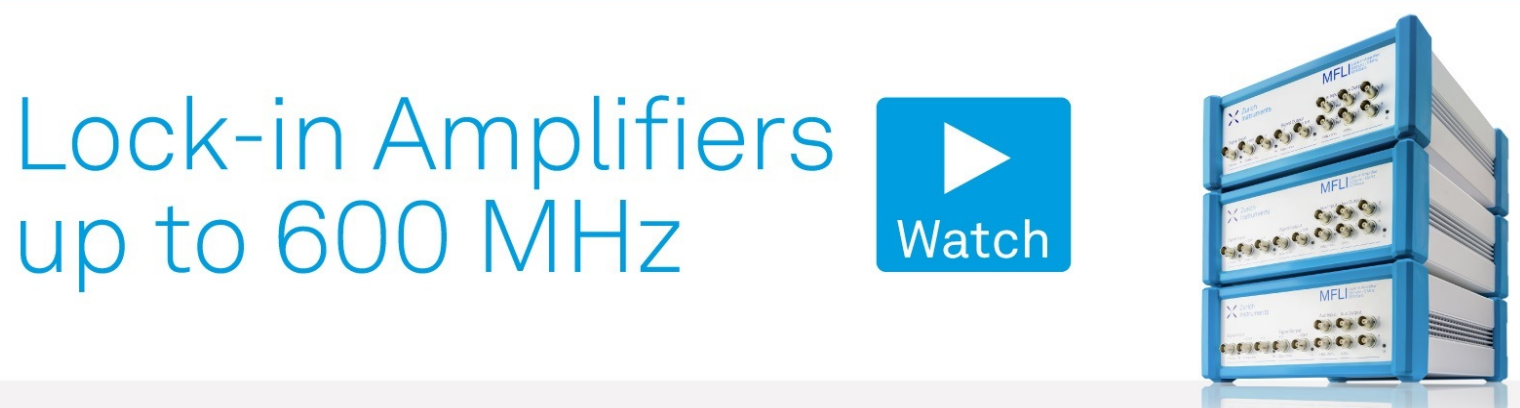


\title{
High-performance planar-type electron source based on a graphene-oxide-semiconductor structure
}

Cite as: Appl. Phys. Lett. 114, 213501 (2019); doi: 10.1063/1.5091585

Submitted: 4 February 2019 - Accepted: 9 May 2019 .

Published Online: 30 May 2019

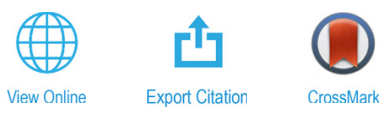

Katsuhisa Murakami, ${ }^{1,2, a)}$ (iD Joji Miyaji, ${ }^{1,3}$ Ryo Furuya, ${ }^{1,4}$ Manabu Adachi, ${ }^{1,2}$ Masayoshi Nagao, ${ }^{1}$ (D Yoichiro Neo, Yoshinori Takao, ${ }^{5}$ (D) Yoichi Yamada, ${ }^{2}$ (D) Masahiro Sasaki, ${ }^{2}$ and Hidenori Mimura ${ }^{3}$

\begin{abstract}
AFFILIATIONS
${ }^{7}$ National Institute of Advanced Industrial Science and Technology, 1-1-1 Umezono, Tsukuba, Ibaraki 305-8568, Japan

${ }^{2}$ Faculty of Pure and Applied Sciences, University of Tsukuba, 1-1-1 Tennodai, Tsukuba, Ibaraki 305-8573, Japan

${ }^{3}$ Research Institute of Electronics, Shizuoka University, 3-5-1 Johoku Naka-ku, Hamamatsu 432-8011, Japan

${ }^{4}$ Department of Mechanical Engineering, Materials Science, and Ocean Engineering, Yokohama National University, 79-5 Tokiwadai, Hodogaya-ku, Yokohama 240-8501, Japan

${ }^{5}$ Division of Systems Research, Yokohama National University, 79-5 Tokiwadai, Hodogaya-ku, Yokohama 240-8501, Japan

${ }^{a)}$ Electronic mail: murakami.k@aist.go.jp
\end{abstract}

\begin{abstract}
A graphene-oxide-semiconductor (GOS) planar-type electron source was fabricated by direct synthesis of graphene on an oxide layer via low-pressure chemical vapor deposition. It achieved a maximum electron emission efficiency of $32.1 \%$ by suppressing the electron inelastic scattering within the topmost gate electrode using a graphene electrode. In addition, an electron emission current density of $100 \mathrm{~mA} / \mathrm{cm}^{2}$ was observed at an electron emission efficiency of $16.2 \%$. The electron energy spread was well fitted to Maxwell-Boltzmann distribution, which indicates that the emitted electrons are the thermally equilibrium state within the electron source. The full-width at half-maximum energy spread of the emitted electrons was approximately $1.1 \mathrm{eV}$. The electron emission efficiency did not deteriorate after more than $42 \mathrm{~h}$ of direct current operation. Thus, the GOS planar-type electron source has the potential to be an excellent electron gun for electron microscopy.
\end{abstract}

Published under license by AIP Publishing. https://doi.org/10.1063/1.5091585

Electron microscopes are widely utilized in materials science, ${ }^{1}$ life sciences, ${ }^{2}$ and semiconductor manufacturing. ${ }^{3}$ One of the key technologies that affect microscope performance is the electron source. Although several materials such as carbon nanotubes ${ }^{4}$ and nanowires $^{5-8}$ have been proposed for electron sources, there are only four types of commercially available sources: tungsten hairpins, $\mathrm{LaB}_{6}$ crystals, ${ }^{9,10}$ tungsten field emitters, ${ }^{11}$ and Schottky emitters. ${ }^{12}$ The thermionic guns made of tungsten hairpins or $\mathrm{LaB}_{6}$ crystals have the advantages of being relatively inexpensive and operable in relatively low vacuum conditions. However, the energy spread of the thermionic electron beam is typically around $2 \mathrm{eV},{ }^{13}$ which causes large chromatic aberration. Furthermore, the brightness of a thermionic electron gun is low because of its large source size (tens of micrometers). These are the main reasons for the relatively low resolution of electron microscopes that have thermionic electron guns. Tungsten fields and Schottky emitters are based on field emission although Schottky emitters operate at high temperatures. These electron sources have advantages of high brightness and narrower energy spreads of $0.3-1.0 \mathrm{eV},{ }^{13}$ which enable a high microscope resolution. However, these sources require a $10^{-8} \mathrm{~Pa}$ high vacuum, which leads to higher instrument costs because of the need for turbomolecular and ion pumps. Therefore, the development of an electron source with a narrow energy spread that is operable in a low vacuum would reduce the cost of high-resolution electron microscopes.

Planar-type electron sources based on a metal-oxide-semiconductor (MOS) structure can be operated at low vacuum, low voltage, and room temperature conditions, ${ }^{14-17}$ and emit electron beams with small divergence angles. ${ }^{18}$ Although these features are advantageous for several applications, such as low-cost, high-resolution electron microscopes, highly sensitive image sensors, ${ }^{19}$ field emission displays, ${ }^{20}$ and electron beam lithography, ${ }^{21,22}$ they have a very low electron emission efficiency of $0.002 \%{ }^{16}$ Therefore, there is insufficient 
electron beam current from an emission area equivalent to the electron source sizes of thermionic electron guns and field-emission guns. The main reason for the low MOS emission efficiency is electron energy loss from inelastic scattering within the oxide and metal electrode. When the electric field is applied to the oxide layer, the width of the potential barrier between the Si substrate and the oxide layer decreases. Then, the electrons at the conduction band of the Si substrate tunnel through the potential barrier and travel through the conduction band of the oxide layer under the electric field. Electrons are scattered and lose their energy at that time. Electrons reached at the interface between the oxide layer and the metal gate are partly reflected by the elastic backscattering. The remaining electrons travel through the metal gate electrode and further lose their energy by inelastic scattering. Most of the electrons that reached the surface of the gate electrode have lower energy than the work function of the gate metal. These low energy electrons are eventually collected by the gate electrode and flow as the gate current. As a result, electron emission efficiency of the MOS type emission device becomes very low. The basic strategy to improve the electron emission efficiency of the MOS devices is the suppression of electron scattering and reduction of the work function of the gate electrode. Another solution is utilizing the cascade tunneling of the electrons within the insulating layer such as $\mathrm{SiO}_{\mathbf{x}}{ }^{23,24}$ and nanocrystal silicon. ${ }^{25,26}$ The emission efficiency of $28 \%$ has been reported using the insulating layer of $\mathrm{SiO}_{\mathrm{x}} \cdot{ }^{23}$ In these device cases, the energy spread of the emitted electrons is around $10 \mathrm{eV}$ in full-width at half-maximum (FWHM), ${ }^{24}$ which is broader than that of the thermal electron source. This is because the larger electron scattering occurs within the insulating layer due to the thicker insulating layer of several hundred nanometers. The electron inelastic scattering cross section of the material generally becomes larger with the atomic number and lower electron energy. In the case of the MOS type electron emission device, the energy of electrons within the device is around $10 \mathrm{eV}$, which induces large electron inelastic scattering. Therefore, the material which has a small electron inelastic scattering cross section should be used as the gate electrode. In addition, the thickness of the gate electrode should be thinner than the electron mean free path of the gate material. Graphene is the atomic layered carbon material with high electrical conductivity. Its electron inelastic scattering cross section is smaller than that of the conventional metal gate electrode such as $\mathrm{Au}$ and Al. These excellent features of graphene make it an ideal material for the gate electrode of the MOS type electron emission source. In fact, the efficiency of planar-type electron sources has been improved to $0.3 \%-13 \%$ by suppression of electron scattering using the graphene gate electrode. $^{27-32}$ However, further improvement of the electron emission efficiency of the planar-type electron emission source has been required to obtain sufficient electron beam current from an emission area equivalent to the electron source sizes of the conventional electron guns. The maximum transmittance of low energy electrons with respect to the $\mathrm{c}$-axis direction of single layer graphene was reported to be around $60 \%{ }^{33}$ This suggests that the maximum electron emission efficiency of a graphene-oxide-semiconductor (GOS) structure is probably around $60 \%$. In the previous reports, the graphene gate electrode was fabricated by transferring the CVD graphene on a $\mathrm{Cu}$ foil. These graphene electrodes have cracks and wrinkles. ${ }^{34,35}$ In addition, it is very difficult to maintain cleanliness at the interface between the insulator and the graphene electrode since the transferring processes are a dirty wet process using resist polymers and the ferric chloride acid solution. These issues cause leakage current and electron traps in the emission devices. Here, improvement of the emission efficiency up to $32.1 \%$ was achieved with a graphene-oxide-semiconductor (GOS) planar-type electron source fabricated by direct synthesis of graphene on the oxide layer via low-pressure chemical vapor deposition (LPCVD). Hence, a GOS planar-type electron source would be a good electron gun candidate for low-cost, high-performance electron microscopes. The performance as an electron source was also characterized.

The fabrication of GOS electron sources is as follows. A highly doped n-type Si substrate $(0.01 \Omega$-cm) with a 300 -nm-thick thermal oxide was used. Electron emission areas of $10 \mu \mathrm{m} \times 10 \mu \mathrm{m}$, $50 \mu \mathrm{m} \times 50 \mu \mathrm{m}$, and $100 \mu \mathrm{m} \times 100 \mu \mathrm{m}$ were fabricated using conventional photolithography and wet etching. A 10 -nm-thick oxide layer was then grown by thermal oxidation at $900^{\circ} \mathrm{C}$ after standard RCA cleaning. Graphene electrodes with thicknesses of $1.0 \mathrm{~nm}$ (3 layers of graphene) and $2.5 \mathrm{~nm}$ (7-8 layers of graphene) were directly synthesized on the entire surface of the substrate by LPCVD at $900^{\circ} \mathrm{C}$ using $\operatorname{Ar}(20 \mathrm{sccm}) / \mathrm{CH}_{4}(1 \mathrm{sccm})$ gas for 1 and $3 \mathrm{~h}$, respectively. Figure 1 shows a schematic of the LPCVD system. The graphene electrode was deposited in the second furnace with respect to the gas flow direction. $\mathrm{CH}_{4}$ was cracked in the first furnace at $1050^{\circ} \mathrm{C}$ before graphene growth in the second furnace. The pressure during graphene growth was maintained at $5000 \mathrm{~Pa}$ by a conductance control valve. The shape of the graphene electrode of the electron sources was patterned by photolithography. Then, graphene uncovered by the photoresist was etched by an $\mathrm{O}_{2}$ plasma for device isolation. $\mathrm{A} \mathrm{Ni} / \mathrm{Ti}$ contact electrode was then fabricated using conventional photolithography, electron beam evaporation, and a lift-off process. An optical and a scanning electron microscopy (SEM) image of a GOS electron source and a Raman spectrum of the graphene electrode are shown in Fig. 2. The graphene electrode of the emission area had no cracks and wrinkles like graphene transferred from the $\mathrm{Cu}$ foil. The $\mathrm{G}$ and $2 \mathrm{D}$ peaks in the spectrum were attributed to the crystal structure of graphene, while the large D peak was attributed to defects in the graphene. ${ }^{36,37}$ These data indicated that the graphene electrode was polycrystalline. The electron emission characteristics were measured in a $10^{-6}$-Pa vacuum chamber evacuated by a turbomolecular pump. A metal-plate anode for applying a voltage of $1 \mathrm{kV}$ was placed $5 \mathrm{~mm}$ away from the gate electrode. The GOS electron sources were annealed at $300^{\circ} \mathrm{C}$ for $1 \mathrm{~h}$ in a $10^{-4}$-Pa load-lock chamber before measurements to clean the surface of the graphene electrode. ${ }^{23}$ The cathode, gate, and anode current were individually measured by the three source measure units, as shown in Fig. 3. The electron emission efficiency was defined as the ratio of anode current to cathode current. The current density is calculated by dividing the anode current by the electron emission area of the device structure. It was already confirmed that the electron emission occurs at the whole area of the electron source. ${ }^{28}$ Therefore, the designed emission area can be considered as the real emission area.

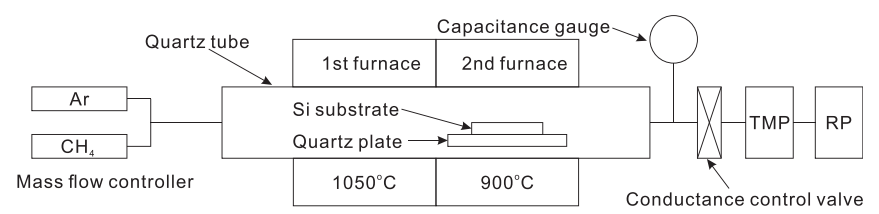

FIG. 1. Schematic of LPCVD equipment for graphene synthesis. 

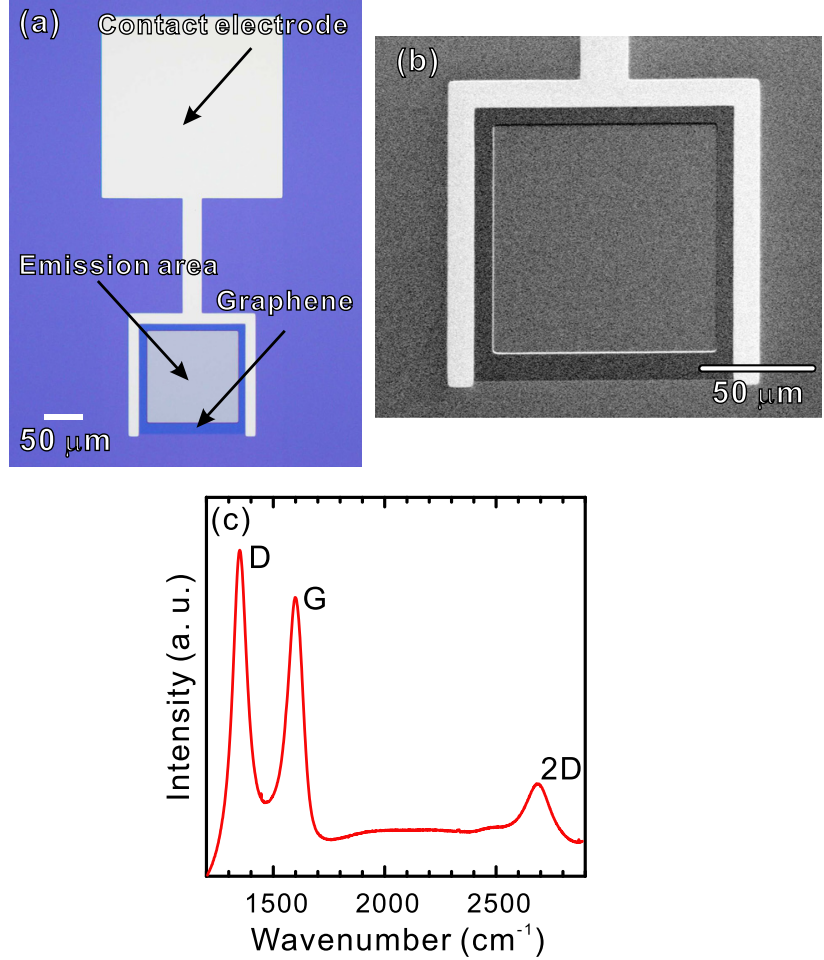

FIG. 2. (a) Optical image of the GOS electron source. (b) SEM image of the emission area of the GOS electron source. (c) Raman spectrum of the graphene electrode.

The possible factor of the error in the calculation of current density is the error of the emission area patterning by photolithography and wet etching. This error is approximately $+300 \mathrm{~nm}$ due to the isotropic wet etching, which leads to the error of approximately $+2.4 \%$ in the case of $50 \mu \mathrm{m}$ square of the emission area. The energy distribution of the emitted electrons from the GOS structure was acquired using a hemispherical electron energy analyzer.

Figure 4(a) shows the emission current density, cathode current density, and electron emission efficiency of the GOS electron source with 1.0-nm-thick graphene as a function of gate bias voltage.

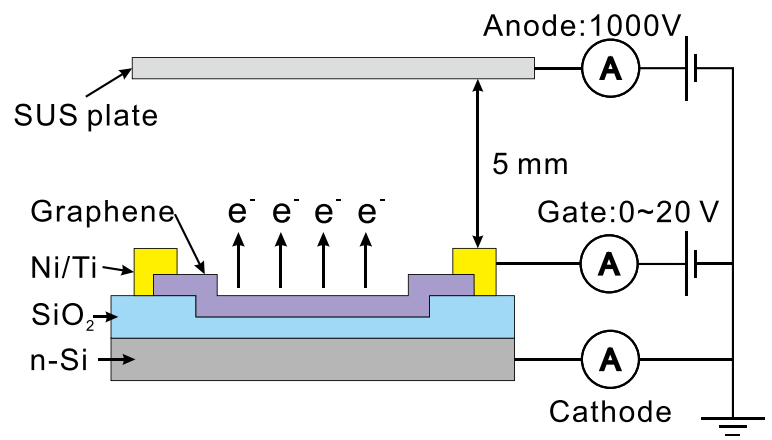

FIG. 3. Schematic of the measurement setup of the electron emission properties of GOS electron sources.
Emission current was detected at a 7-V gate bias and reached $96 \mu \mathrm{A} /$ $\mathrm{cm}^{2}$ at $9 \mathrm{~V}$. The emission efficiency had a maximum of $32.1 \%$ at $8.1 \mathrm{~V}$ and remained almost constant at higher gate biases. In addition, a current density of $100 \mathrm{~mA} / \mathrm{cm}^{2}$ was observed with an emission efficiency of $16.2 \%$ in another electron source, as shown in Fig. 4(b). The measured resistivity of the graphene electrode and the contact resistivity of the $\mathrm{Ni} / \mathrm{Ti} /$ graphene junction are $5.29 \times 10^{-6} \Omega \mathrm{m}$ and $1.7 \times 10^{5} \Omega \mu \mathrm{m}$, respectively. The contact resistances of the electron emission source with an emission area size of $10 \mu \mathrm{m} \times 10 \mu \mathrm{m}, 50 \mu \mathrm{m} \times 50 \mu \mathrm{m}$, and $100 \mu \mathrm{m} \times 100 \mu \mathrm{m}$ calculated by using the contact resistivity and the contact length of the metal electrode for each electron sources are $1.7 \mathrm{k} \Omega, 772 \Omega$, and $459 \Omega$, respectively. On the other hand, the maximum cathode current level of the electron sources is in the range of several nanoamperes for $10 \mu \mathrm{m} \times 10 \mu \mathrm{m}$ to several tens of microamperes for $100 \mu \mathrm{m} \times 100 \mu \mathrm{m}$. Therefore, the contact resistance does not affect the measurement of the electron emission properties of the electron source.

Figure 4(c) shows the electron emission efficiency as a function of the electron emission current density for GOS electron sources with graphene thicknesses of $1.0 \mathrm{~nm}$ and $2.5 \mathrm{~nm}$. For the 2.5-nm-thick graphene, the efficiency was in the range of $2 \%-7 \%$. In contrast, the efficiency exceeded $10 \%$ for all electron sources having 1.0 -nm-thick graphene. These results indicate that the electron transmittance of graphene increases with the decrease in its thickness. In addition, several electron sources showed the almost same electron emission properties of the emission current density of around $100 \mathrm{~mA} / \mathrm{cm}^{2}$ with an emission efficiency of around $16 \%$, which indicates the good reproducibility of their performance. Both the electron emission efficiency and the emission current density of the GOS electron sources were four orders of magnitude higher than those of conventional MOS-type electron sources. The current density of $100 \mathrm{~mA} / \mathrm{cm}^{2}$ corresponded to a beam current of $2 \mu \mathrm{A}$ from the $50-\mu \mathrm{m}$-diameter electron emission area that was comparable to the source size of $\mathrm{LaB}_{6}$ crystal. $^{13}$ This beam current level was sufficient for electron microscopy because of the low divergence angle from a planar-type electron source and if most of the electron beam passes through the electron optics and reaches the sample.

Figure 5 plots the electron energy distribution of the GOS electron source with 1.0-nm-thick graphene at a gate bias of $9.06 \mathrm{~V}$. The origin of the electron energy was the Fermi level of the Si substrate, and the FWHM of the energy spread was approximately $1.1 \mathrm{eV}$. The energy distribution was well fitted to Maxwell-Boltzmann distribution, which indicates that the emitted electrons were largely scattered within the oxide layer and became the thermally equilibrium state in the conduction band of the oxide at the near interface between the oxide and the electrode. Therefore, the energy distribution of the emitted electrons from the graphene based planar type electron emission source is similar to that of thermal-type cathodes rather than that of field emission type cathodes. However, the energy spread of the GOS electron sources was narrower than the 2-eV spread of tungsten hairpin and $\mathrm{LaB}_{6}$ crystal thermal-type cathodes in electron microscopes. ${ }^{13}$ Therefore, the GOS electron source potentially has a microscope performance between that of the $\mathrm{LaB}_{6}$ cathode and the Schottky emitter.

Figure 6 shows the time dependence of emission current density, cathode current density, and electron emission efficiency of the GOS type electron source with a $2.5-\mathrm{nm}$-thick graphene thickness at a gate bias of $10 \mathrm{~V}$. The emission current density and cathode current density 

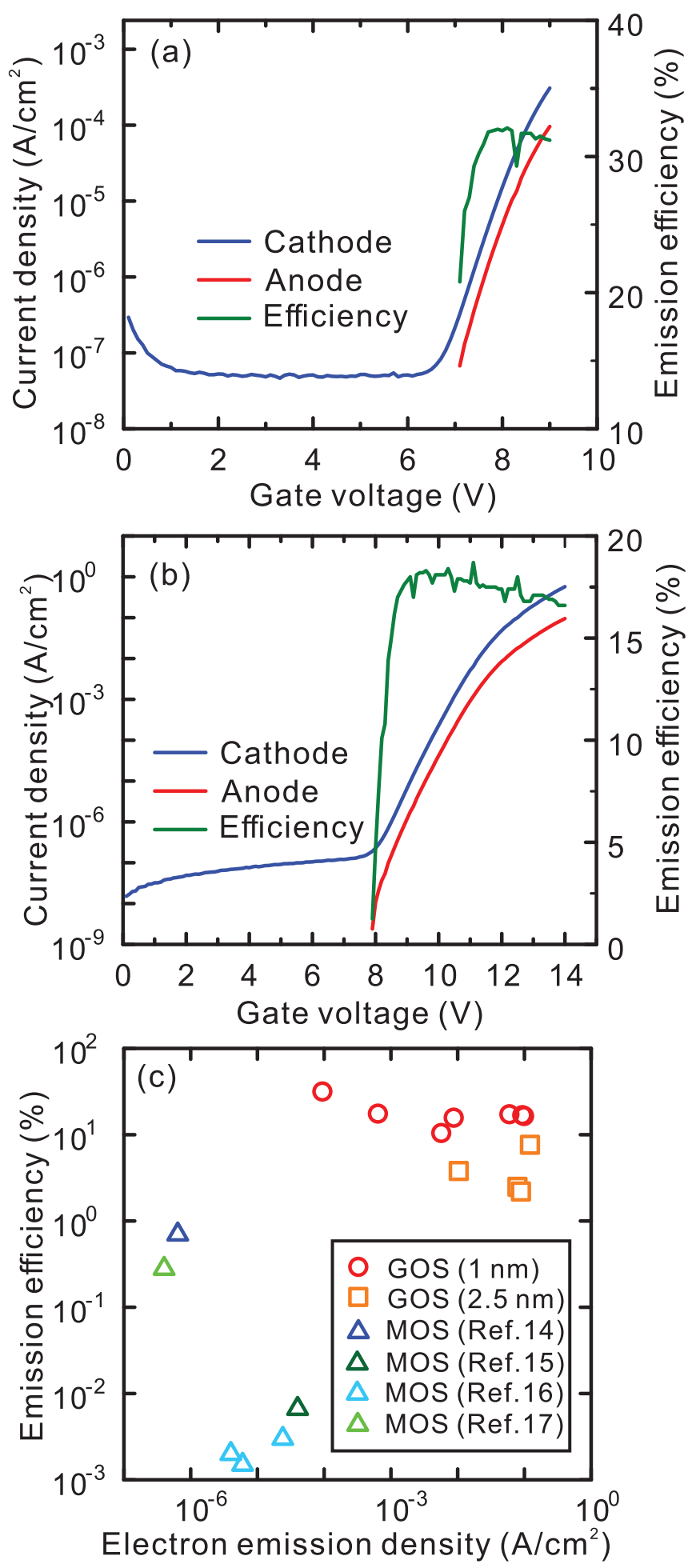

FIG. 4. (a) and (b) Emission current density, cathode current density, and electron emission efficiency of the GOS electron source with 1.0-nm-thick graphene vs gate bias voltage. (c) Electron emission efficiencies of GOS electron sources having graphene thicknesses of $1.0 \mathrm{~nm}$ and $2.5 \mathrm{~nm}$ vs electron emission current density.

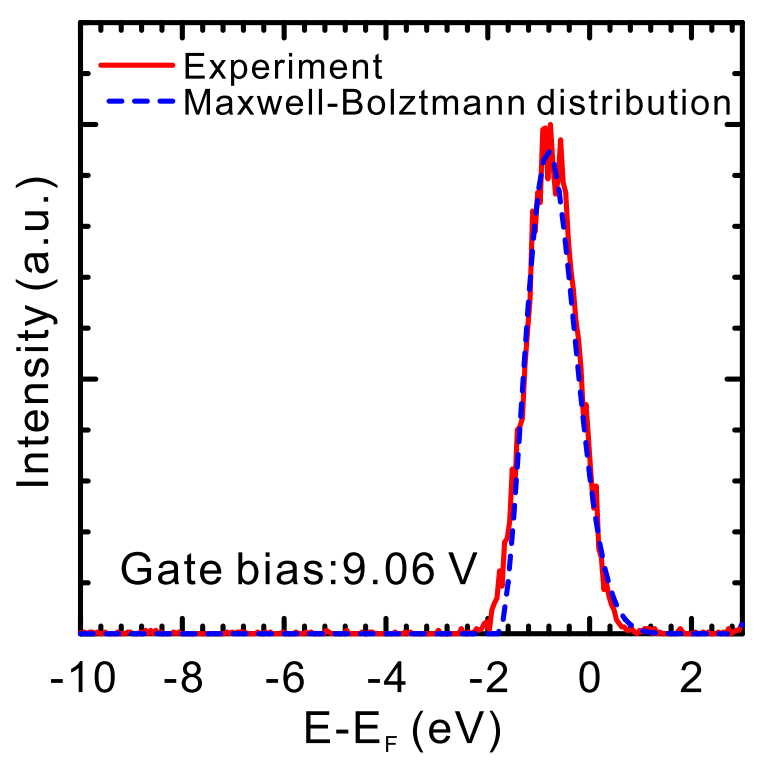

FIG. 5. Energy distribution of electrons emitted from a GOS electron source having 1.0 -nm-thick graphene at a gate bias of $9.06 \mathrm{~V}$.

gradually decreased simultaneously. However, the electron emission current density remained at a higher level of $35 \mu \mathrm{A} / \mathrm{cm}^{2}$ after being driven by $42 \mathrm{~h}$ of direct current. In addition, the electron emission efficiency was a constant value of $5 \%$ for more than $42 \mathrm{~h}$. This current level is higher than the specification of the prototype of the parallel electron beam lithography system $\left(10 \mu \mathrm{A} / \mathrm{cm}^{2}\right),{ }^{22}$ which is enough for several applications of the electron beams although further improvement of the current density is required. The total amount of charge passing through the oxide layer was approximately $160 \mathrm{C} / \mathrm{cm}^{2}$ without the dielectric breakdown of the oxide layer. This value exceeded the typical $Q_{\mathrm{DB}}$ (charge-to-breakdown) of a MOS device, ${ }^{38}$ even though the GOS oxide layer thickness and the graphene electrode thickness were not optimized. Thus, the lifetime of the GOS electron source

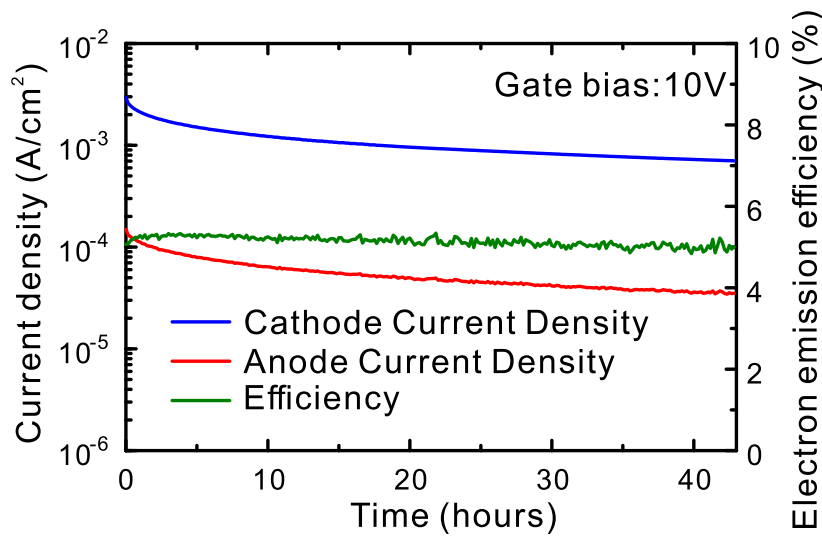

FIG. 6. Time dependence of the emission current density, cathode current density, and electron emission efficiency of a GOS electron source with 2.5 -nm-thick graphene at a gate bias of $10 \mathrm{~V}$. 
could be longer than that of conventional MOS-type sources. A detailed evaluation of the lifetime of the GOS electron source, including time-dependent dielectric breakdown, will be reported elsewhere.

In summary, high-performance electron sources based on a GOS structure fabricated by direct LPCVD synthesis of graphene were demonstrated. A maximum electron emission efficiency of $32.1 \%$ was observed. In addition, an electron emission current density of $100 \mathrm{~mA} / \mathrm{cm}^{2}$ was observed with an efficiency of $16.2 \%$. The FWHM energy spread of the electron beams emitted from the GOS structure was $1.1 \mathrm{eV}$, which was narrower than that of tungsten hairpin and $\mathrm{LaB}_{6}$ crystal thermionic cathodes. Overall, these results indicate that the GOS planar-type electron source has great potential as an electron gun for a low-cost, high-resolution electron microscope operating at low vacuum.

This work was partly supported by the Ministry of Education, Culture, Sports, Science and Technology (MEXT) of Japan through a Grant-in-Aid for Young Scientists (A) (Grant No. 15H05522), a Grant-in-Aid for Challenging Exploratory Research (Grant No. 16K14223), a Grant-in-Aid for Scientific Research (B) (Grant No. 18H01505), and a Grant-in-Aid for Challenging Research (Exploratory) (Grant No. 18K18910) and by Nanotech Career-up Alliance (Nanotech CUPAL). This work has been done as part of JAXA/RDD's active space-debris removal research. Support was also received from the NIMS Molecule and Material Synthesis Platform, the NIMS Microstructural Characterization Platform, the AIST Nanofabrication Platform, and the University of Tsukuba Nanofabrication Platform in the Nanotechnology Platform Project sponsored by MEXT, Japan.

\section{REFERENCES}

'Z. L. Wang, J. Phys. Chem. B 104, 1153 (2000).

${ }^{2}$ Y. Sugita, H. Matsunami, Y. Kawaoka, T. Noda, and M. Wolf, Nature 563, 137 (2018).

${ }^{3}$ R. S. Rai and S. Subramanian, Prog. Cryst. Growth Charact. Mater. 55, 63 (2009).

${ }^{4}$ W. A. de Heer, A. Châtelain, and D. Ugarte, Science 270, 1179 (1995).

${ }^{5}$ Z. Pan, H. L. Lai, F. C. K. Au, X. Duan, W. Zhou, W. Shi, N. Wang, C. S. Lee, N. B. Wong, S. T. Lee, and S. Xie, Adv. Mater. 12, 1186 (2000).

${ }^{6}$ C. C. Chen, C. C. Yeh, C. H. Chen, M. Y. Yu, H. L. Liu, J. J. Wu, K. H. Chen, L. C. Chen, J. Y. Peng, and Y. F. Chen, J. Am. Chem. Soc. 123, 2791 (2001).

${ }^{7}$ C. J. Lee, T. J. Lee, S. C. Lyu, Y. Zhang, H. Ruh, and H. J. Lee, Appl. Phys. Lett. 81, 3648 (2002)

${ }^{8}$ H. Zhang, J. Tang, Q. Zhang, G. Zhao, G. Yang, J. Zhang, O. Zhou, and L. C. Qin, Adv. Mater. 18, 87 (2006).

${ }^{9}$ A. N. Broers, J. Appl. Phys. 38, 1991 (1967).

${ }^{10}$ A. N. Broers, J. Appl. Phys. 38, 3040 (1967).
${ }^{11}$ A. V. Crewe, D. N. Eggenberger, J. Wall, and M. Welter, Rev. Sci. Instrum. 39, 576 (1968).

${ }^{12}$ L. W. Swanson and N. A. Martin, J. Appl. Phys. 46, 2029 (1975).

${ }^{13}$ A. Bogner, P. H. Jouneau, G. Thollet, D. Basset, and C. Gauthier, Micron 38, 390 (2007).

${ }^{14}$ K. Yokoo, H. Tanaka, S. Sato, J. Murota, and S. Ono, J. Vac. Sci. Technol., B 11, 429 (1993).

${ }^{15}$ K. Yokoo, S. Sato, G. Koshita, I. Amano, J. Murota, and S. Ono, J. Vac. Sci. Technol., B 12, 801 (1994).

${ }^{16}$ K. Yokoo, G. Koshita, S. Hanzawa, Y. Abe, and Y. Neo, J. Vac. Sci. Technol., B 14, 2096 (1996).

${ }^{17}$ H. Mimura, Y. Abe, J. Ikeda, K. Tahara, Y. Neo, H. Shimawaki, and K. Yokoo, J. Vac. Sci. Technol., B 16, 803 (1998).

${ }^{18}$ H. Shimawaki, Y. Neo, H. Mimura, K. Murakami, F. Wakaya, and M. Takai, J. Vac. Sci. Technol., B 26, 864 (2008).

${ }^{19}$ N. Negishi, T. Sato, Y. Matsuba, R. Tanaka, T. Nakada, K. Sakemura, Y. Okuda, A. Watanabe, T. Yoshikawa, K. Ogasawara, M. Nanba, S. Okazaki, K. Tanioka, N. Egami, and N. Koshida, J. Vac. Sci. Technol., B 26, 711 (2008).

${ }^{20}$ T. Kusunoki, M. Suzuki, M. Sagawa, Y. Mikami, E. Nishimura, M. Ikeda, T. Hirano, and K. Tsuji, J. Vac. Sci. Technol, B 30, 041202 (2012).

${ }^{21}$ M. Poppeller, E. Cartier, and R. M. Tromp, Appl. Phys. Lett. 73, 2835 (1998).

${ }^{22}$ M. Esashi, A. Kojima, N. Ikegamim, H. Miyaguchi, and N. Koshida, Microsyst. Nanoeng. 1, 15029 (2015).

${ }^{23}$ N. Negishi, T. Chuman, S. Iwasaki, T. Yoshikawa, H. Ito, and K. Ogasawara, Jpn. J. Appl. Phys., Part 2 36, L939 (1997).

${ }^{24}$ K. Sakemura, N. Negishi, T. Yamada, H. Satoh, A. Watanabe, T. Yoshikawa, and K. Ogasawara, J. Vac. Sci. Technol., B 22, 1367 (2004).

${ }^{25}$ X. Sheng, H. Koyama, and N. Koshida, J. Vac. Sci. Technol., B 16, 793 (1998).

${ }^{26}$ K. Nishiguchi, X. Zhao, and S. Oda, J. Appl. Phys. 92, 2748 (2002).

${ }^{27}$ C. Wenger, J. Kitzmann, A. Wolff, M. Fraschke, C. Walczyk, G. Lupina, W. Mehr, M. Junige, M. Albert, and J. W. Bartha, J. Vac. Sci. Technol., B 33, $01 \mathrm{~A} 109$ (2015).

${ }^{28}$ K. Murakami, S. Tanaka, A. Miyashita, M. Nagao, Y. Nemoto, M. Takeguchi, and J. Fujita, Appl. Phys. Lett. 108, 083506 (2016).

${ }^{29}$ K. Nishiguchi, D. Yoshizumi, Y. Sekine, K. Furukawa, A. Fujiwara, and M. Nagase, Appl. Phys. Express 9, 105101 (2016).

${ }^{30}$ M. P. Kirley, T. Aloui, and J. T. Glass, Appl. Phys. Lett. 110, 233109 (2017).

${ }^{31}$ K. Murakami, S. Tanaka, T. Iijima, M. Nagao, Y. Nemoto, M. Takeguchi, Y. Yamada, and M. Sasaki, J. Vac. Sci. Technol., B 36, 02C110 (2018).

${ }^{32}$ A. Kojima, R. Suda, and N. Koshida, Appl. Phys. Lett. 112, 133102 (2018).

${ }^{33}$ G. Hassink, R. Wanke, I. Rastegar, W. Braun, C. Stephanos, P. Herlinger, J. H. Smet, and J. Mannhart, APL Mater. 3, 076106 (2015).

${ }^{34}$ A. Reina, H. Son, L. Jiao, B. Fan, M. Dresselhaus, Z. Liu, and J. Kong, J. Phys. Chem. C 112, 17741 (2008).

${ }^{35}$ A. Srivastava, C. Galande, L. Ci, L. Song, C. Rai, D. Jariwala, K. F. Kelly, and P. M. Ajayan, Chem. Mater. 22, 3457 (2010).

${ }^{36}$ F. Tuinstra and J. L. Koenig, J. Chem. Phys. 53, 1126 (1970).

${ }^{37}$ A. C. Ferrari, J. C. Meyer, V. Scardaci, C. Casiraghi, M. Lazzeri, F. Mauri, S. Piscanec, D. Jiang, K. S. Novoselov, S. Roth, and A. K. Geim, Phys. Rev. Lett. 97, 187401 (2006).

${ }^{38}$ P. P. Apte, T. Kubota, and K. C. Saraswat, J. Electrochem. Soc. 140, 770 (1993). 